\title{
DETERMINATION OF TECHNICAL AND ECONOMIC INDICATORS OF THERMAL POWER STATIONS DIRECTLY FROM THE TURBINE REGIME CHARACTERISTICS
}

\author{
Konstantin Kostov \\ Technical University of Sofia \\ Faculty of Engineering and Pedagogy of Sliven \\ 59 Burgasko Shose blvd., Sliven, Bulgaria, 8800 \\ kostov_77@abv.bg
}

\begin{abstract}
The distribution of costs for electricity and heat production is accomplished by a number of fairly conditional methods. Cost analysis and allocation must be carried out on the basis of objective technical and economic criteria. The application of a method is determined by the introduced regulatory frameworks, energy markets and the prices of energy products. There are two fundamental methods - "physical" and "exergy". The physical method is based on the distribution of costs, and the exergy on the second law of thermodynamics. The article reviews and analyzes the tariff policy of the Thermal power plant. Objective criteria have been identified to serve as a means of forecasting and controlling fuel consumption. The thermal efficiency of a Thermal power plant has been evaluated. A comparison was made with similar Thermal power plants. A fundamentally new approach is proposed to determine the main technical and economic criteria of the plant. The proposed method allows determining indicators that uniquely characterize the thermal and economic efficiency of the plant without the need for diversification of fuel costs.
\end{abstract}

Keywords: Thermal power plant, Ginter's triangle, physical method, tariffs, economic efficiency.

DOI: $10.21303 / 2461-4262.2020 .001358$

\section{Introduction}

Determination of the indicators of thermal and economic efficiency of thermal power plants and as a consequence of the formation of tariffs for electricity and heat, in combined energy production, in purely mathematical terms, it is a simple Diophantine equation [1,2], one unknown, which is on determined in advance depending on physical, technical or economic criteria, as appropriate. Already at the beginning of the development of thermal power plants, a clear method for visualization of solutions, called the Ginter's triangle, was developed - Fig. 1, [3-5]. If the Cartesian coordinates of the ordinate axis represent the costs of electricity production and the abscissa axis of heat, there are two extreme positions in which one component is the maximum and the other zero. The straight line between them forms in the first quadrant a right-angled triangle with the origin of the coordinate system. As the total cost does not change, all distribution options are the working points lying on the hypotenuse. The orthogonal projection of each operating point on the coordinate axes defines a single solution with two values for electricity and heat. When allocating costs to a third product, such as desalinated water, additional technical and economic criteria must be analysed as a cost allocation tool to determine the contribution of each product to the total production cost [6-8]. Then the Ginter's triangle transforms and becomes a pyramid. The operating points are already in the plane and the solutions are their projections on the three axes. Numerous options for solving the problem of diversification of fuel costs are essentially reduced to the application of one of the two fundamental methods known as "physical" and "exergy". The physical method is based on the distribution of costs, in proportion to the amount of fuel consumed for each type of energy on the basis of heat balance, while the exergy method of the second law of thermodynamics, characterizing the quality of different types of energy. The main difference between the two methods of analysis is that, is that the exergy takes into account, not so much the quantitative equivalents of energy flows, but the energy losses during its transformation. In their developments a number of authors [9-15] have made sufficiently in-depth analyses and comparisons of both approaches, as well as of the hybrid "Energy" method, also known as the "ORGRES method" $[16,17]$. All known methods of fuel distribution and the subsequent costs between electricity and heat are proving to be equally conditional and should be applied with great care. They give completely different results and therefore no one of them can serve as a basis for shap- 
ing the tariff policy of thermal power plants under market conditions. The experience of the leading countries in the European Union, the legal framework introduced [18] and the analysis carried out [19] convince us of the need to apply exclusively economic mechanisms, by abandoning attempts to bind pricing solely with the problems of distribution fuel on TPP.

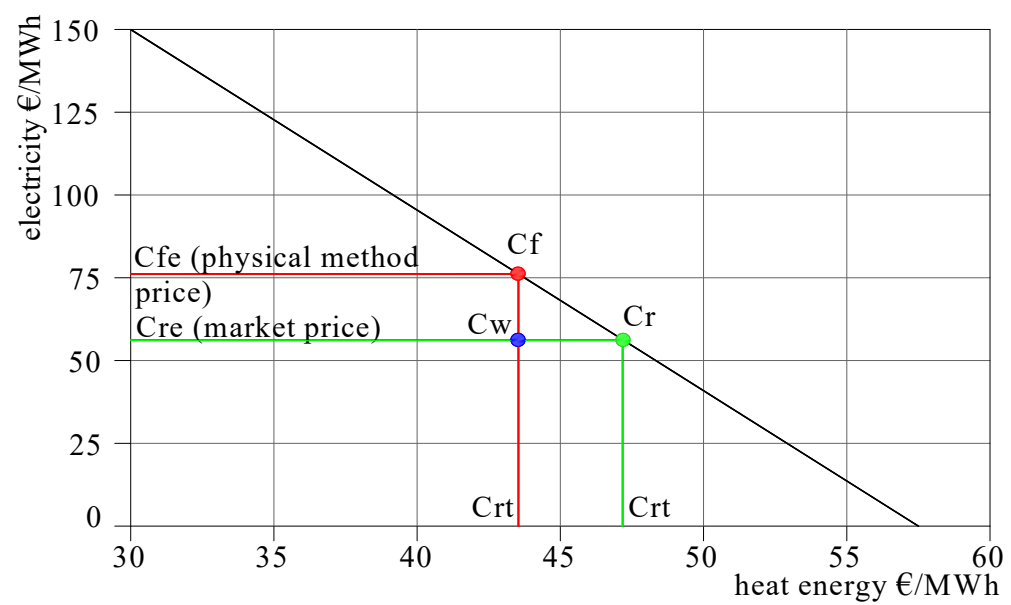

Fig. 1. Ginter's triangle

The object of the study is the TPP at an oil refinery. The plant was put into operation in 1963 , after which it was gradually expanded and modernized. In terms of composition and technology, he has the characteristic features of his time. Designed to operate on a combined basis, the electrical load is determined predominantly based on the thermal consumption of the plant.

The technical and economic indicators and the energy tariffs are determined by the distribution of the fuel for the respective type of production, by the imposed normative physical method. With the privatization of the refinery and the switchover and in private hands, the conditions under which the power plant operated significantly changed. Due to the changes, the energy capacities were separated from the refinery in an independent energy enterprise with the activity of extraction of electricity, heat and desalinated water. From the very beginning, the question arose of setting fair rates at which the new company would sell its products. Although the Energy and Water Regulatory Commission sets the tariffs for industrial unit plant thermal power plants and district heating companies using the same methodology $[20,21]$ there is a significant difference between them. While National Electric Company is obliged to buy electricity from district heating companies, even though it has no economic interest, nothing can oblige factories to buy electricity from their former power plants at certain prices, provided that twice as cheap energy is available on the market. The difficulties in operating the market conditions of the plant are further compounded by the sharp decline in the oil refinery's thermal loads, due to the limitation of the volume of main production and the management's deliberate policy to reduce the heat consumption in technological installations [24]. The economic survival of the thermal power plant necessitates an unconditional restriction of electricity production to the possible technical minimum in accordance with the new heat loads [25].

This, in turn, results in the exploitation of out-of-date and spent main and auxiliary equipment in technological regimes far from the optimal ones with increased costs.

The purpose of the study is instead of the "physical" method, to determine the technical and economic characteristics of the TPP directly from the operating characteristics of the turbine. The advantages of the proposed method are outlined and the tariff policy of the plant when operating under market conditions is analysed.

\section{Methodology of the study}

The purpose of this work is to analyse the tariff policy of the oil refinery at market conditions and to propose a fundamentally new approach for determining the main technical and economic indicators of the plant without diversifying fuel costs. 
Two tasks arise from the stated goal. The first task is to determine objective technical and economic indicators to serve as a means of forecasting, normalizing and controlling fuel consumption, as well as criteria for evaluating thermal efficiency when compared to similar plants. The second task is to find market-based mechanisms for shaping the tariff policy of the plant.

The application of any variant for dividing the total cost of fuel by type of production leads to the solution of the first task, with the proviso that each method implies the expediency of using it. Considering the fact that only one turbine is required to cover the loads PT-50-130/555, a new approach is proposed that is based on the machine's operating characteristics. The method allows determining indices that uniquely characterize the thermal and economic efficiency of the plant without the need for diversification of fuel costs.

It is known that the algebraic form of the mode diagram of a turbine with two steam extractions, depending on the electrical $(W)$, industrial $\left(D_{P}\right)$ and district heating load $\left(D_{T}\right)$ is of the type:

$$
D=d_{0}+d_{E} W+y_{P} D_{P}+y_{T} D_{T}
$$

where the coefficients represent respectively the specific costs of fresh steam for idling $d_{0}[\mathrm{t} / \mathrm{h}]$ and production of electricity $d_{\mathrm{E}}[\mathrm{t} / \mathrm{Mwh}]$, as $y_{P}$ and $y_{\mathrm{T}}$ are the coefficients for not using the enthalpy drop respectively for industrial and district heating steam extraction.

By definition:

$$
y_{p}=\frac{\left(i_{p}-i_{k}\right)}{\left(i_{o}-i_{k}\right)}, \quad y_{T}=\frac{\left(i_{T}-i_{k}\right)}{\left(i_{o}-i_{k}\right)},\left[\frac{\mathrm{kJ}}{\mathrm{kg}}\right] \text {. }
$$

The dependences (2) involve the enthalpies of:

- the fresh steam $i_{o}=3,480[\mathrm{~kJ} / \mathrm{kg}]$

- the industrial steam extraction $i_{P}=3,090[\mathrm{~kJ} / \mathrm{kg}]$;

- the district heating $i_{T}=2,700[\mathrm{~kJ} / \mathrm{kg}]$;

- the steam extraction in the capacitor $i_{k}=2,280[\mathrm{~kJ} / \mathrm{kg}]$.

The indicated values of enthalpies depending (2) are average on the basis of the daily work schedules of the plant for the considered period.

At the same time the coefficients of non-use the enthalpy floor is characterized by an increase in steam flow through the turbine $\left(D_{o}-D_{k}\right)$ related to the steam consumption during the respective steam extraction:

$$
y_{p}=\frac{\left(D_{o}-D_{k}\right)}{\left(D_{P}\right)}, y_{T}=\frac{\left(D_{o}-D_{k}\right)}{\left(D_{T}\right)},\left[\frac{\mathrm{kJ}}{\mathrm{kg}}\right] .
$$

In their physical sense, they represent the specific consumption of fresh steam for the heat dissipated through industrial and district heating steam extraction. According to the technical data of the turbine [22] at nominal steam parameters and regeneration included equation (1) is specified:

$$
D=10+3.6 W+0.68 D_{P+} 0.35 D_{T}
$$

It follows from the equation of the straight balance of the steam generator:

$$
B y \cdot H u \cdot \eta=D\left(i_{o}-i_{s w}\right)+D_{p r}\left(i_{H}-i_{s w}\right),
$$

where:

- the enthalpy of steam in the drum $i_{H}=1,550[\mathrm{~kJ} / \mathrm{kg}]$;

- the supply water $i_{s w}=950[\mathrm{~kJ} / \mathrm{kg}]$;

- the lower heat of combustion $H_{u}=29,300[\mathrm{~kJ} / \mathrm{kg}]$;

- the amount of blowdown water $D_{p r}=0,05 \mathrm{D}$.

After substitution with numerical values is obtained ratio: 


$$
\frac{B_{y}}{D}=\frac{(3,480-950)+0.05(1,550-950)}{0.85 \cdot 23,900}=0.13 \text {. }
$$

According to (6), the efficiency ( $\eta$ ) is reduced from 0,9 to 0,85 due to losses in steam lines to turbine shut-off valve and the need to maintain both turbine bypasses in hot reserve. Thus, the conditional fuel consumption $B y,[\mathrm{~kJ} / \mathrm{kg}]$ of the steam turbine installation is:

$$
B y=0.13 D=1.3+0.47 W+0.088 D_{P}+0.046 D_{T}
$$

In equation (7) the coefficients $d_{E}=0.47$ [tCF/Mwh], $y_{P}=0.088[\mathrm{tCF} / \mathrm{t}]$ and $y_{T}=0.046[\mathrm{tCF} / \mathrm{t}]$ are the specific cost of conditional fuel per unit of production, and $d_{0}=1.3[\mathrm{t} / \mathrm{h}]$ conditional fuel consumption for idling the turbine and represent the necessary and sufficient technical and economic criteria for evaluating the efficiency of the steam turbine installation. For uniformity and comparison with the technical and economic indicators normalized by the physical method, the specific costs of conditional fuel $y_{P}=0.088[\mathrm{tCF} / \mathrm{t}]$ and $y_{T}=0.046[\mathrm{tCF} / \mathrm{t}]$ for rendered steam, it is appropriate to recalculate the specific costs of conditional fuel for the released heat - respectively from industrial $Q_{P}[\mathrm{MWh}]$ and district heating $Q_{\mathrm{T}}[\mathrm{MWh}]$ steam extraction:

$$
\begin{aligned}
& Q_{P=} 3.6 \cdot 10^{3} D_{P}\left[i_{P}-a_{b c} \cdot i_{b c}-\left(1-a_{b c}\right) i_{a w}\right], \\
& Q_{P=} 3.6 \cdot 10^{3} D_{T}\left[i_{T}-a_{b c} \cdot i_{b c}-\left(1-a_{b c}\right) i_{a w}\right] .
\end{aligned}
$$

The back condensate is received and qualifies in the TPP, but is not returned to the heat scheme, but is added to the desalinated water and used as process water in the refinery. For this reason, its share in the balance of the plant $a_{b c}=0$ and dependencies (8) are modified in:

$$
\begin{gathered}
Q_{P}=3.6 \cdot 10^{3} D_{P}\left(i_{P}-i_{a w}\right), \\
Q_{T}=3.6 \cdot 10^{3} D_{T}\left(i_{T}-i_{a w}\right) .
\end{gathered}
$$

In enthalpy of the additional water $i_{a w}=126[\mathrm{~kJ} / \mathrm{kg}]$, is obtained:

$$
\begin{aligned}
& D_{P}=1.21 Q_{P}, \\
& D_{T}=1.39 Q_{T} .
\end{aligned}
$$

After the transformations, equation (7) takes the following final form:

$$
B y=0.13 D=11,388+0.47 W+0.1 Q_{P}+0.06 Q_{T}
$$

In fact, the plant consumes more fuel than the formula (11). The reason is that significant both steam extractions are used for internal consumption at the plant, such as keeping a hot reserve on the oil fuel, heating the air in the steam heaters before the boilers, liquid fuel dispersion in burners, for heating and more. The determination of this energy should be carried out by a balance sheet and reported by adding as absolute value to $d_{0}[\mathrm{t} / \mathrm{h}]$, or use "estimated disposable" instead of "lower working" combustion heat of formula (7).

The second task cannot be solved by the traditional method of diversification of fuel consumption. In determining the conditions and arrangements for converting the plant to an independent legal entity, it became apparent that if the plant sells the extracted electricity at prices close to market prices, and the heat at the cost determined by the physical method, due to the high fuel value, the plant would not be able to function as an enterprise because the operating point is obtained below the line of profitability (hypotenuse) of the Ginter's triangle, point $C w$ in Fig. 1. 
Finding a satisfactory solution is possible using the known method of "the split profitability". In the presence of a market price for one product (electricity), a contract is concluded and its sale is carried out without determining the components of its prime cost. The financial result of the conversion is deducted from the total costs (constant and variable), the remainder being related to the production of the other product (heat). In the Ginter's triangle - Fig. 1, this distribution corresponds to $\mathrm{Cr}$. The significant share of desalinated water in the total receipts transforms the task into three-dimensional, with the geometric interpretation changing from a triangle to a pyramid-Fig. 3. Using this approach eliminates the need to separate fuel consumption between electricity Fig. 3. and heat. This is the practice in most countries of the European Union.

Thus, the price of thermal energy $C_{T}[€ / \mathrm{MWh}]$ is determined by the following formula:

$$
C_{T}=\frac{\left(S_{v}+S_{P}\right)-S_{E}-S_{d w}}{Q_{T}},
$$

where the value of variable costs $\left(S_{v}\right)$, fixed costs $\left(S_{p}\right)$, the value of electricity realized $\left(S_{\mathrm{E}}\right)$ and the value of realized water desalinated $\left(S_{d w}\right)$ are measured in $[€]$, and the released heat energy $Q_{T}[\mathrm{MWh}]$.

Obviously:

$$
S_{\mathrm{E}}=C_{\mathrm{E}} W \text { and } S_{d w}=C_{d w} \mathrm{O} .
$$

As $C_{E}[€ / \mathrm{MWh}]$ and $C_{d w}[€ / \mathrm{t}]$ are respectively the price of electricity sold and this desalinated water and $W[\mathrm{MWh}]$ and $O[\mathrm{t}]$ the corresponding quantities for the period considered.

The value of variable costs $S v[€]$ are defined as the sum of the fuel costs $S_{f}[€]$, reagents $S_{R}[€]$ and ancillary resources $S_{A R}[€]$ such as nitrogen, raw water, technical air and more:

$$
S v=S_{f}+S_{R}+S_{A R}
$$

The value of the fuel is also the sum of the costs for the different types of fuels used in the thermal power plant:

$$
S_{f}=S_{b f}+S_{m}+S_{b g}
$$

Here with $S_{b f}[€], S_{m}[€]$ and $S_{b g}[€]$ the costs for: boiler fuel, natural gas and factory fuel gases are indicated.

The fixed costs $S_{P}[€]$ are determined by the formula:

$$
S_{p}=\sum_{k=0}^{n} S_{s}+S_{w i n}
$$

where sums $\left(S_{s}\right)$ of materials not included in variable costs, depreciation, repair costs, interest on loans, etc. are summed up. Add and regulated profit $\left(S_{w i n}\right)$ which should ensure some return on capital.

\section{Results}

To summarize the results and analyse the applicability of the proposed method Fig. 2 presents the prices of the main energy resources for the refinery from the energy market and the supply from TPP for a period of 18 months (June 2018 to February 2020). It is evident that the price of natural gas for the period increased by $28,77 \%$, while the average price of electricity purchased from the free market for the main activities of the refinery during the same period increased by only $3,3 \%$. The price of electricity from the thermal power plant follows this trend and increases only by $2,6 \%$, while the price of heat increases by $54 \%$, practically equalizing with the price of electricity. The price of desalinated water has not changed for the period. 


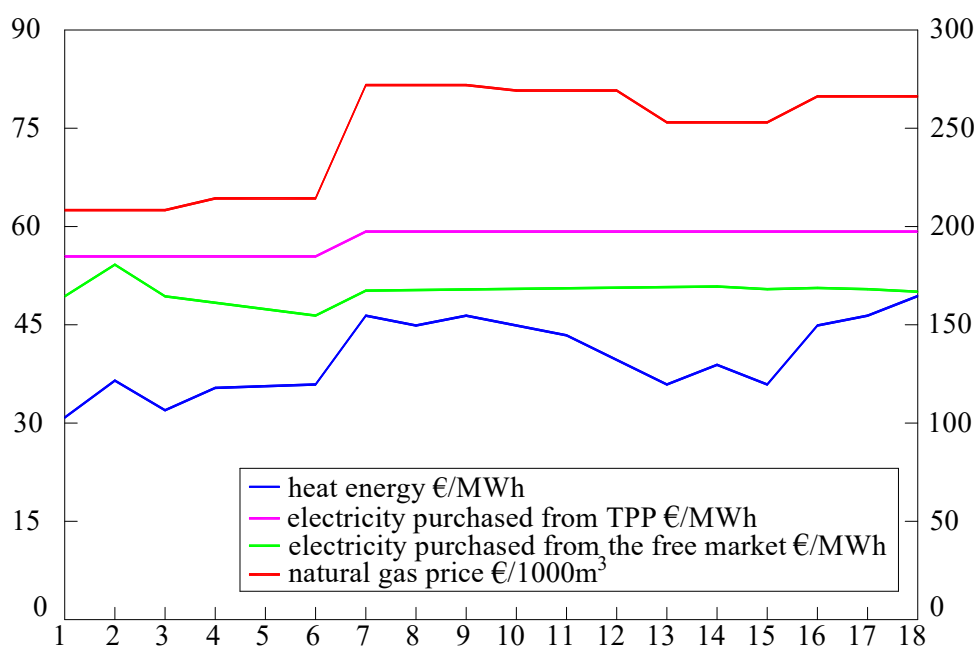

Fig. 2. Change in energy prices

The values of heat and electricity costs obtained in the calculation by the proposed method are compared with the "physical" method. As the method introduces separate specific fuel consumption for the released heat energy for each steam extraction, as well as a specific idle consumption, it is not necessary to introduce summer and winter consumption norms. Thus, the specific fuel consumption for electricity generation no longer depends on the heat load and its seasonal consumption. A comparison of the obtained results with the physical method is shown in Table 1.

Table 1

Comparison of the obtained results with the physical method

\begin{tabular}{cccc}
\hline Indicator & $\begin{array}{c}\text { Definition, } \\
\text { Dimensionality }\end{array}$ & $\begin{array}{c}\text { «Turbine } \\
\text { regime» method }\end{array}$ & «Physical» method \\
\hline $\begin{array}{c}\text { Specific expenditure of conditionally fuel for } \\
\text { electricity generation }\end{array}$ & $d_{E},[\mathrm{tCF} / \mathrm{MWh}]$ & 0,47 & Winter 0,300 \\
Summer 0,326 \\
$\begin{array}{c}\text { Specific expenditure of conditionally fuel for heat } \\
\text { energy generation }\end{array}$ & $\begin{array}{c}y_{P},[\mathrm{tCF} / \mathrm{MWh}] \\
y_{T},[\mathrm{tCF} / \mathrm{MWh}]\end{array}$ & 0,088 & 0,146 \\
$\begin{array}{c}\text { Specific expenditure of conditionally fuel for turbine } \\
\text { operation at idle }\end{array}$ & $d_{0},[\mathrm{t} / \mathrm{h}]$ & 1,3 & -
\end{tabular}

\section{Discussion}

The proposed method for determining thermal efficiency is logical and thermodynamically justified [23]. The results are plausible with regeneration turned on and insignificant deviations from the design fresh steam parameters. The method is more precise than the physical method because instead of a generic one, it introduces for each steam extraction separate specific fuel consumption for the released heat energy, as well as a specific idle expense. The technical and economic indicators thus determined can be easily used for operational planning purposes - it is sufficient to know the expected consumption of electricity and heat for industrial and heating purposes for the period considered. There is no need to introduce summer and winter consumption rates, because the specific fuel consumption for electricity production is no longer dependent on heat load and its seasonal consumption. The parallel operation of another turbine is not economically justified, and even if for some reason, it is short-lived and cannot cause serious inaccuracy. However, if this is a fact for a longer period it is no problem to consider the relative share for each machine in the coefficients of equation (4). The method becomes difficult to apply in parallel operation of more than two different turbines. In this case, the thermodynamic approach presented in [22] seems more acceptable. 
The analysis of the results shows that the determination of the cost price and, accordingly, the cost of the heat energy of formula (8) have some fundamental disadvantages:

- all contingent fixed costs incl. depreciation, staff salaries, repairs and more at the Electro factory and the Chemical factory, which have nothing to do with heat production, are an integral part of the cost of this product;

- all costs for reagents relate to heat, while only a small fraction of them (hydrazine, sodium phosphate) are related to its production. In the same wrong way, only the costs of auxiliary resources, such as nitrogen technical, instrumentation, air, raw water, et al are written off in the heat energy alone;

- the cost of own electricity of the entire plant indirectly (through fuel) is also calculated in the cost of heat energy.

Fig. 3 shows the Ginter's modified triangle of pyramid constructed with summarized data for the period.

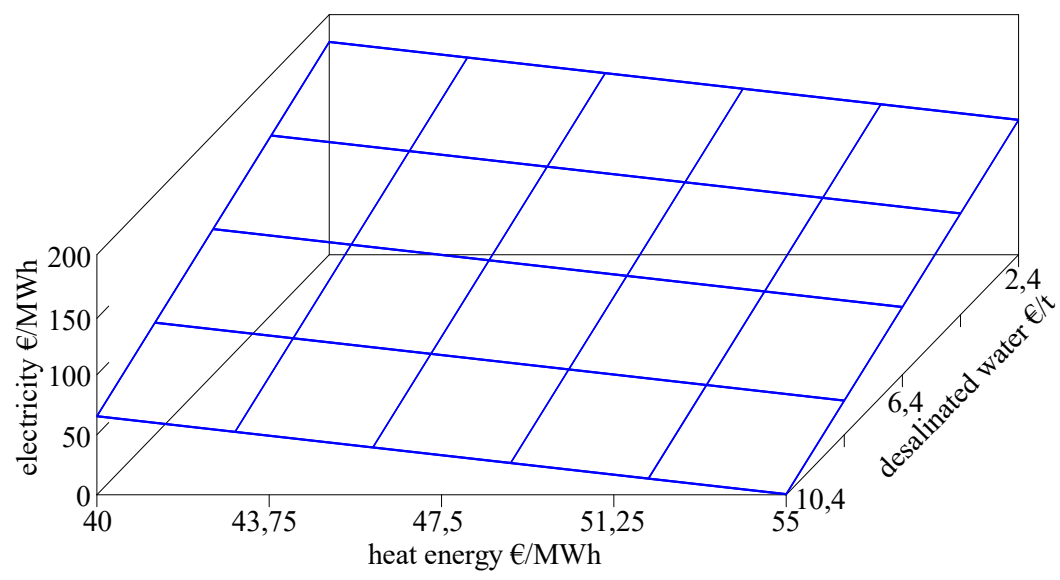

Fig. 3. Modified Ginter's triangle in pyramid

\section{Conclusion}

The general conclusion from the study that can be made is that the equalization of the prices of heat and electricity is thermodynamically unjustified. Buying expensive fuel and keeping the sale price of electricity leads to an increase in the price of heat. The economic conditions under which the plant operates require the cost of heat to be determined solely by the costs associated with its production. In the absence of funds for manufacturing activity, other means of financing need to be sought. The implementation of the proposed method gives credible results that can be used in the operation of the plant. Because the method is based on the diagram of the PT-50-130/555 turbine, it has significant advantages over the physical method.

\section{Acknowledgements}

The author/s would like to thank the Research and Development Sector at the Technical University of Sofia for the financial support.

\section{Nomenclature}

$B y$ - Conditional fuel consumption.

$C_{T}$ - Price of thermal energy.

$D_{P}$ - Industrial load.

$D_{T}$ - Heating load.

$H_{u}$ - The lower heat of combustion.

$Q_{P}$ - Industrial steam extraction.

$Q_{T}-$ District heating steam extraction.

TPP - Thermal power plant.

$W$ - Electrical load. 


\section{References}

[1] Ribenboim, P. (1986). Some Fundamental Methods in the Theory of Diophantine Equations. Aspects of Mathematics and Its Applications, 635-663. doi: https://doi.org/10.1016/s0924-6509(09)70286-7

[2] Brüdern, J., Dietmann, R. (2014). Random Diophantine equations, I. Advances in Mathematics, 256, 18-45. doi: https:// doi.org/10.1016/j.aim.2014.01.017

[3] Tubolev, A., Romashova, O., Belyaev, L. (2016). Cost Price of Products in the System of Heat, Refrigeration and Electric Energy Production Combined at Thermal Power Plant. MATEC Web of Conferences, 72, 01116. doi: https://doi.org/10.1051/ matecconf/20167201116

[4] Zharkov, S. (2007). Fuel consumption separation for heat and electricity produced by TEC. Gas Turbine Technologies, 11, $34-40$.

[5] Koryakin, Y. I., Loginov, A. A., Chernyaev, V. A. (1970). Methodological aspects of technico-economic parameters of nuclear desalination plants. Desalination, 7 (3), 323-342. doi: https://doi.org/10.1016/s0011-9164(00)80205-2

[6] Catrini, P., Cipollina, A., Micale, G., Piacentino, A., Tamburini, A. (2017). Exergy analysis and thermoeconomic cost accounting of a Combined Heat and Power steam cycle integrated with a Multi Effect Distillation-Thermal Vapour Compression desalination plant. Energy Conversion and Management, 149, 950-965. doi: https://doi.org/10.1016/j.enconman.2017.04.032

[7] Tamburini, A., Cipollina, A., Micale, G., Piacentino, A. (2016). CHP (combined heat and power) retrofit for a large MED-TVC (multiple effect distillation along with thermal vapour compression) desalination plant: high efficiency assessment for different design options under the current legislative EU framework. Energy, 115, 1548-1559. doi: https://doi.org/10.1016/j.energy.2016.03.066

[8] Piacentino, A. (2015). Application of advanced thermodynamics, thermoeconomics and exergy costing to a Multiple Effect Distillation plant: In-depth analysis of cost formation process. Desalination, 371, 88-103. doi: https://doi.org/10.1016/j.desal.2015.06.008

[9] Sukhareva, E. (2015). Cost allocation methods when forming cost of energy for CHP. Transport business in Russia, 2, $43-45$.

[10] Lisin, E. M., Stepanova, T. M., Zhovtiak, P. G. (2017). Investigation of the effect of cost allocation methods on the competitiveness of CHP plants in energy markets. St. Petersburg State Polytechnical University Journal. Economics, 256 (6), $148-158$. doi: https://doi.org/10.5862/je.256.13

[11] Franco, A., Bellina, F. (2018). Methods for optimized design and management of CHP systems for district heating networks (DHN). Energy Conversion and Management, 172, 21-31. doi: https://doi.org/10.1016/j.enconman.2018.07.009

[12] Franco, A., Versace, M. (2017). Multi-objective optimization for the maximization of the operating share of cogeneration system in District Heating Network. Energy Conversion and Management, 139, 33-44. doi: https://doi.org/10.1016/j.enconman.2017.02.029

[13] Ege, A., Şahin, H. M. (2014). Determination of uncertainties in energy and exergy analysis of a power plant. Energy Conversion and Management, 85, 399-406. doi: https://doi.org/10.1016/j.enconman.2014.05.088

[14] Erdem, H. H., Akkaya, A. V., Cetin, B., Dagdas, A., Sevilgen, S. H., Sahin, B. et. al. (2009). Comparative energetic and exergetic performance analyses for coal-fired thermal power plants in Turkey. International Journal of Thermal Sciences, 48 (11), 2179-2186. doi: https://doi.org/10.1016/j.ijthermalsci.2009.03.007

[15] Denisov, V. (2001). Method for the formation of tariffs for electric and thermal energy. Thermal Engineering, 4, 58-61.

[16] Methodical instructions for drawing up a report of the power plant and the joint-stock company of energy and electrification on the thermal economy of equipment RD 34.08.552-95 (1995). Moscow: ORGRES.

[17] Zamaleev, M. M., Gubin, I. V., Sharapov, V. I., Bushuev, E. N. (2018). Calculation methods of power efficiency of combined heat and power plant at change of equipment operating modes and thermal schemes. Journal of Physics: Conference Series, 1111, 012039. doi: https://doi.org/10.1088/1742-6596/1111/1/012039

[18] Directive 2004/8/EC of the European Parliament and of the Council of 11 February 2004 on the promotion of cogeneration based on a useful heat demand in the internal energy market and amending Directive 92/42/EEC.

[19] Hrilev, L., Malafeev, B., Haraim, A., Livchic, I. (2003). Comparative assessment of domestic and foreign methods for distributing fuel consumption and setting tariffs for TPP. Thermal Engineering, 4, 45-54.

[20] Ordinance No. RD-16-267 of 19 March 2008; on the determination of the amount of electricity produced from the combined production of heat and electricity, Ministry of Economy and Energy of the Republic of Bulgaria.

[21] Ganev, P. (2009). Bulgarian electricity market restructuring. Utilities Policy, 17 (1), 65-75. doi: https://doi.org/10.1016/j.jup. 2008.02.005

[22] Piir, A. E., Kuntysh, V. B. (2006). Determination of indexes of thermal and economic efficiencies of a cogeneration power plant without dividing fuel consumption and the equipment between two kinds of plant production. Thermal Engineering, 53 (5), 399-402. doi: https://doi.org/10.1134/s0040601506050120

[23] Fallahi, A., Ebrahimi, R., Ghaderi, S. F. (2011). Measuring efficiency and productivity change in power electric generation management companies by using data envelopment analysis: A case study. Energy, 36 (11), 6398-6405. doi: https:// doi.org/10.1016/j.energy.2011.09.034 
[24] Nikolov, Ch., Shishmanov, Iv., Krystev, N., Angelova, D. (2014). Attempting to predict the specific heat consumption in installation hydro desulphurisation of gasoline in "Lukoil Neftochim Burgas" AD. Thermal engineering, 5 (3), 18-23.

[25] Nikolov, Ch., Atanasov, K. (2013). Some results of the energy cost survey and energy consumption trends of "Lukoil Neftochim Burgas" AD. XVIII Scientific conference with international participation fpepm 2013, Proceedings, 1, $221-223$.

\title{
IMPROVEMENT OF OPERATION MODES OF THE EVAPORATOR OF THE ABSORPTION REFRIGERATING UNIT
}

\author{
Oleksandr Titlov ${ }^{1}$ \\ titlov1959@gmail.com \\ Daniyorbek Adambayev ${ }^{1}$ \\ adambayev90@gmail.com \\ Oleg Vasyliv ${ }^{1}$ \\ oleg_vas@ukr.net \\ ${ }^{1}$ Department of Heat-and-Power Engineering and Fuel Pipeline Transportation \\ Odessa National Academy of Food Technologies \\ 112 Kanatna str., Odessa, Ukraine, 65039
}

\begin{abstract}
Absorption refrigeration units (ARU), which are part of absorption refrigeration devices (ARD) with a natural working fluid (water, ammonia and hydrogen) have a number of unique qualities. These qualities include: noiselessness, high reliability and long life; the possibility of using several energy sources in one device. At the same time, ARDs have increased energy consumption compared to similar compression models, and this does not allow them to expand their presence in the market of household refrigeration equipment.

The ARU evaporator provides a predetermined temperature level in the chambers of the refrigeration appliance and the required cooling capacity. In this regard, it is relevant to search for the operating modes of the evaporator that provide the ARU maximum energy efficiency, which is the aim of this work.

The thermal conditions of the direct-flow three-pipe design of the evaporator are simulated. The calculated ratio for a oncethrough evaporator is obtained taking into account the assumption of the adiabaticity of the evaporation process, when all the heat of the phase transition is used to cool the incoming flows of the purified vapor-gas mixture (VGM) and liquid ammonia to a minimum temperature.

The analysis of the results of calculating the operating modes of the evaporator made it possible to determine the directions of ways to increase the energy efficiency of both the evaporator itself and the ARU in general:

a) preliminary cooling of the purified VGM flow at the inlet of the adiabatic section of the evaporator with an under-recovery of up to $5^{\circ} \mathrm{C}$ and up to $10^{\circ} \mathrm{C}$;

b) preliminary cooling of the liquid ammonia flow at the inlet of the adiabatic section of the evaporator with an under-recovery of up to $5^{\circ} \mathrm{C}$ for all ARU types;

c) increasing the purification degree of the VGM flow in the absorber allows increasing the temperature of the purified VGM flow at the inlet of the adiabatic section of the evaporator by $4 \ldots 6^{\circ} \mathrm{C}$, i. e. to reduce the costs of useful cooling capacity for pre-cooling by $10 \ldots 15 \%$.
\end{abstract}

Keywords: absorption refrigerating devices and units, evaporator, heat and mass transfer, energy saving. 